Methods PBMCs were isolated and we first determined monocytes subpopulations and looked at expression of membrane TNF (mTNF). After negative selection of monocytes, Macrophages were Derived from Monocytes(MDM) by 7 days of culture in the presence of M-CSF (M2 differentiation) or GM-CSF (M1 differentiation). Expression of total macrophages markers (CD11b and CD71) and the M2 macrophage polarisation markers (CD163 and CD206) were evaluated.

Results We have confirmed that the $\mathrm{CD} 14^{+} \mathrm{CD} 16^{+}$monocytes subset was expanded in RA patients. For the first time, we have demonstrated that mTNF expression was significantly increased only in monocytes in RA patients $\left(\mathrm{CD} 14^{+}\right.$: Mean 5.6\% HD versus $10.6 \% \mathrm{RA}, \mathrm{CD} 14^{+} \mathrm{CD} 16^{+}$: Mean 3.3\% HD versus 11.1\% RA, CD16 ${ }^{+}$: Mean 2.4\% HD versus 6.3\% RA). Moreover, mTNF expression on monocytes correlated with the activity of the disease assessed by DAS28 CRP (Spearman $r \quad 0.548$ and $\left.p=0.0021^{*}\right)$. We have observed a significant decrease of macrophages induction by M-CSF in RA patients as shown by a decreased expression of CD11b-CD71(Mean 87.2\% HD versus $57.1 \%$ RA, P value $* * * *)$. Among MDM, we have found a specific decreased level of M2 markers (CD206 Mean 78.1\% HD versus 27\% RA and CD163 $56.2 \%$ HD versus $37.2 \%$ RA) suggesting an impaired maturation to M2 stage in RA patients.

Conclusions Monocytes from RA patients have an increased expression of mTNF linked to activity of the diseases. RA patients have an impaired maturation of monocytes to M2 macrophages. This might suggest that RA monocytes have a propensity for preferential maturation towards a pro-inflammatory M1 phenotype thus contributing to synovial inflammation. Deeper characterisation of $\mathrm{M} 1 / \mathrm{M} 2$ and effect of the different types of anti-TNF on this differentiation process are on-going and will be presented.

Disclosure of interest None declared

\section{P022 CHECKPOINT INHIBITORS ACTIVATE STORE-OPERATED CA2+ ENTRY AND ERK1/2 SIGNALLING AND PROMOTE TH17 DIFFERENTIATION}

B Zapp*, P Lehmkuhl, H Schulze-Koops, A Skapenko. Sektion Rheumatologie und Klinische Immunologie, Medizinische Klinik und Poliklinik IV, Universität München, Munich, Germany

\subsection{6/annrheumdis-2018-EWRR2018.47}

Introduction Anti-tumour therapy with immune checkpoint inhibitors for programmed-death 1 receptor (PD1), such as nivolumab is often accompanied by immune-related adverse events (irAE). The cellular and molecular mechanisms underlying this phenomenon are not defined yet. Interaction of PD-1 with its ligand (PD-L1) mediates potent inhibitory signals to hinder proliferation and effector function of $\mathrm{T}$ cells. Interruption of PD1:PD-L1 interaction by nivolumab during antitumour therapy might therefore amplify $\mathrm{T}$ cell receptor (TCR) signalling and facilitate the development of a pro-inflammatory autoimmune response.

Objectives To investigate the impact of PD1 inhibition on intracellular signalling mechanisms down-stream of the TCR such as calcium $\left(\mathrm{Ca}^{2+}\right)$ influx and activation of mitogen-activated protein kinases (MAPK) pathway and to assess the effect of PD1 inhibition on $\mathrm{T}$ cell effector function by evaluating the cytokine profile of the nivolumab-treated $\mathrm{T}$ cells.

Methods CD4 memory $\mathrm{T}$ cells were stimulated with anti-CD3 and anti-CD28 in the presence of nivolumab for 24 hour.
Afterwards intracellular $\mathrm{Ca}^{2+}$ influx in response to ionomycin was assessed by flow cytometry following loading the cells with Fluo-8. Expression of proteins involved in store-operated $\mathrm{Ca}^{2+}$ entry (SOCE), stromal activation molecule (STIM) 1, an activator of $\mathrm{Ca}^{2+}$ release-activated $\mathrm{Ca}^{2+}$ (CRAC) channels, and Orai1, a component of CRAC channels was determined by TaqMan real-time PCR. Phosphorylation of extracellular signal-regulated kinase 1 and 2 (Erk1/2) in response to antiCD3/28 was determined by intracellular flow cytometry. The cytokine profile of nivolumab-treated cells was assessed after four-day culture by intracellular flow cytometry.

Results Treatment of CD4 $\mathrm{T}$ cells with nivolumab led to a pronounced increase of the ionomycin-mediated $\mathrm{Ca}^{2+}$ influx. At the same time expression of SOCE proteins, STIM1 and Orai1, was significantly up regulated in the nivolumab-treated cells. Phosphorylation of Erk1/2 in response to short antiCD3/CD28 restimulation was almost twice as high in CD4 T cells cultured with as without nivolumab. Finally nivolumabtreated cells contained higher frequencies of IL-17-producing T cells (Th17 cells).

Conclusions Interruption of PD1:PD-L1 interaction by nivolumab activate SOCE and promotes Erk1/2 activation. Both $\mathrm{T}$ cell signalling pathways are essential for a proper mounting an immune response. Their deregulation might therefore precede an abnormal $\mathrm{T}$ cell response as shown for example by increased Th17 cell frequency and facilitate the onset of autoimmune phenomena such as irAE.

Acknowledgements This work was supported by DFG grants SK59/09-1 and Schu1683/10-1, and by BMBF Project Arthromark 01EC1401B.

Disclosure of interest None declared

\section{P023 SMOKING IS ASSOCIATED WITH LOW SERUM LEVELS OF SOLUBLE PD-L1 IN RHEUMATOID ARTHRITIS}

${ }^{1} \mathrm{C}$ Wasén*, ${ }^{1} \mathrm{M}$ Erlandsson, ${ }^{2,3,4} \mathrm{~A}$ Bossios, ${ }^{2} \mathrm{~L}$ Ekerljung, ${ }^{2} \mathrm{C}$ Malmhäll, ${ }^{1} \mathrm{~S}$ Töyrä Silfverswärd, ${ }^{1} \mathrm{R}$ Pullerits, ${ }^{2} \mathrm{~B}$ Lundbäck, ${ }^{1} \mathrm{Ml}$ Bokarewa. ${ }^{1}$ Department of Rheumatology and Inflammation Research; ${ }^{2}$ The Krefting Research Centre, Department of Internal Medicine and Clinical Nutrition, University of Gothenburg, Gothenburg; ${ }^{3}$ Department of Respiratory Medicine and Allergy, Karolinska University Hospital; ${ }^{4}$ Department of Medicine, Karolinska Institutet, Huddinge, Sweden

\subsection{6/annrheumdis-2018-EWRR2018.48}

Introduction Smoking is a risk factor for the development of rheumatoid arthritis (RA) and associates with positivity for disease specific anti cyclic citrullinated peptide (aCCP) antibodies. It is not known exactly how smoking promotes autoimmunity but we have previously demonstrated that smoking limits the expression of the $\mathrm{T}$ cell co-inhibitory receptor programmed death-1 (PD-1). ${ }^{1}$

Objectives To investigate if smoking can interfere with the interaction between PD-1 and its ligand by influencing the serum levels of soluble PD-1 ligand 1 (sPD-L1).

Methods Serum samples were collected from 254 RA patients and 168 healthy controls with known smoking status and analysed with ELISA for levels of sPD-L1 and inflammatory cytokines IL-6 and IL-1b. Fc-receptor mRNA expression analysis of peripheral blood monocytes (PBMC) from a group of 10 healthy controls and 15 RA patients was done by qPCR.

Results In RA patients current smokers had 5 times lower median serum levels of sPD-L1 compared to never smokers $(\mathrm{p}=0.027)$. This difference persisted in former smokers that quit smoking $<25$ years ago $(\mathrm{p}=0.0086)$. Expectedly, sPD-L1 
were associated with high serum levels of inflammatory markers IL-6 (over $5.5 \mathrm{pg} / \mathrm{ml}, \mathrm{p}=0.0006$ ) and IL-1b (over $4 \mathrm{pg} / \mathrm{ml}, \mathrm{p}=0.0005$ ), however the level of these markers was not affected by the smoking status of the patients. We found 14 times lower sPD-L1 levels in smoking RA patients that did not receive TNF-inhibitors $(p=0.0092)$, but treatment with TNF-inhibitors normalised levels of sPD-L1.

Furthermore, aCCP positivity in RA patients was associated with higher levels of sPD-L1 $(p=0.0036)$. We speculate that the presence of antibodies might influence the levels of sPDL1 through the stimulation of Fc-receptors expressed by PDL1 producing cells. In PBMC depleted of T cells, we saw that smokers had lower mRNA expression of the stimulatory FcgRIIIA $(p=0.028)$ and predominance of the inhibitory FcgRIIB in the FcgRIIB/FcgRIIIA ratio $(p=0.0004)$.

Conclusions Smoking decreases the serum levels of the inflammation limiting protein sPD-L1, but levels were restored by treatment with TNF-inhibitors. aCCP positive RA patients had higher levels of sPD-L1, possibly due to activation of Fcreceptors expressed by PD-L1 producing cells.

\section{REFERENCE}

1. Wasén C, et al. J Autoimmun 2017.

Disclosure of interest None declared

\section{P024 COMPARISON OF CCP2 AND CCP3 ASSAYS IN A LARGE COHORT OF ESTABLISHED RHEUMATOID ARTHRITIS AND CONTROLS}

${ }^{1} \mathrm{M}$ Malher, ${ }^{1} \mathrm{C}$ Bentow, ${ }^{1} \mathrm{R}$ Albesa, ${ }^{1} \mathrm{~L}$ Cesana, ${ }^{1} \mathrm{~L}$ Martinez-Prat, ${ }^{2} \mathrm{P}$ Roux-Lombard, ${ }^{3}$ MJ Nissen, ${ }^{3} \mathrm{C}$ Lamacchia*, ${ }^{3} \mathrm{C}$ Gabay. ${ }^{1}$ INOVA Diagnostics, Inc., San Diego, USA; ${ }^{2}$ Division Immunology-Allergy; ${ }^{3}$ Division of Rheumatology, University Hospital Geneva, Geneva, Switzerland

\subsection{6/annrheumdis-2018-EWRR2018.49}

Introduction Rheumatoid Factor (RF) and anti-citrullinated protein antibodies (ACPA) are important serological marker in the diagnosis of rheumatoid arthritis (RA) and are part of the classification criteria. ACPA are generally detected using anticyclic citrullinated peptide (CCP) antibody assays. The first generation of the CCP test uses a peptide derived from the filaggrin protein as the antigen, whereas, the second and third generation CCP (CCP2, CCP3) are based on peptides specifically designed and optimised (mimotypes) to detect ACPA, thereby enhancing the immunoreactivity of the citrulline-containing epitope.

Objectives The goal was to compare the performance of CCP2 and CCP3 assays.

Methods 1655 samples including 968 RA patients and 687 controls (450 ankylosing spondylitis (AS) and 237 psoriatic arthritis (PsA) patients), all derived from the Swiss Clinical Quality Management in Rheumatic Diseases Foundation (SCQM) were included. ACPA were determined by CCP2 ELISA (Eurodiagnostica, Sweden), CCP3 ELISA (QUANTA Lite CCP3 IgG) and CCP3 CIA (QUANTA Flash CCP3 IgG) (both Inova Diagnostics, US). RF IgM was measured by ELISA (QUANTA Lite RF IgM, Inova Diagnostics, US).

Results The CCP2 ELISA showed a high sensitivity (71.1\%) and a moderately high specificity $(86.9 \%)$ with a corresponding Odds ratio (OR) of 16.3 (95\% CI: 12.5 to 21.1). The two CCP3 assays showed lower sensitivities $(61.8 \%$ for the ELISA and $61.4 \%$ for the CIA), but significantly higher specificities (98.4\% and $98.5 \%$ respectively), resulting in much higher predictive values, with OR of 99.3 (95\% CI: 54.4 to 181.2) and 107.5 (95\% CI: 57.4 to 201.5), respectively. When compared at the same specificity (95\%), the sensitivities were $61.3 \%$ for the CCP2 ELISA, $68.1 \%$ for the CCP3 ELISA and $66.1 \%$ for the CCP3 CIA. When multi-parametric analyses were performed by combining ACPA with RF IgM, combining different markers resulted in higher OR than the individual markers. The combination of CCP3 and RF IgM resulted in a higher $\mathrm{OR}(\mathrm{OR}=187.0,510 / 1655)$ than the combination of CCP2 with RF IgM (OR=36.7, 565/1655). The addition of CCP2 to the combination of CCP3 and RF IgM resulted in a lower OR $(\mathrm{OR}=175.0,494 / 1655)$.

Conclusions CCP3 showed a better overall performance than CCP2 in this cohort of RA and controls, when analysed individually as well as in combination with RF IgM.

Disclosure of interest None declared

\section{P025 THE DIAGNOSTIC AND PROGNOSTIC VALUE OF AUTOANTIBODIES IN RHEUMATOID ARTHRITIS}

${ }^{1} \mathrm{D}$ Sieghart*, 'A Platzer, 'F Alasti, ${ }^{1} \mathrm{P}$ Studenic, ${ }^{2} \mathrm{M}$ Grundhuber, ${ }^{2} \mathrm{~S}$ Swiniarski, 'S Blueml, ${ }^{3}$ T Perkmann, ${ }^{1} \mathrm{~J}$ Smolen, ${ }^{1} \mathrm{G}$ Steiner. 'Department of Internal Medicine III, Division of Rheumatology, Medical University of Vienna, Vienna, Austria; ${ }^{2}$ ImmunoDiagnostic Division, Thermo Fisher Scientific, Phadia GmbH, Freiburg, Germany; ${ }^{3}$ Department of Laboratory Medicine, Medical University of Vienna, Vienna, Austria

\subsection{6/annrheumdis-2018-EWRR2018.50}

Introduction Rheumatoid factor (RF) and anti-citrullinated protein antibodies (ACPA) are important diagnostic tools in rheumatoid arthritis (RA). These antibodies are predominantly of the $\operatorname{IgM}(\mathrm{RF})$ or IgG (ACPA) isotype. Other isotypes of both antibodies - like IgA - and other antibodies - like anti-RA33, which is directed to the nuclear antigen hnRNP-A2 - have been repeatedly reported but their diagnostic and prognostic value has still not been fully elucidated.

Objectives Here we investigated (i) the prevalence of IgA-RF and IgA-ACPA as well as isotypes $\operatorname{IgA}$, IgG and $\operatorname{IgM}$ of antiRA33 antibody in patients with RA and (ii) their predictive value regarding therapeutic response to methotrexate (MTX).

Methods Sera from 290 RA patients, 261 disease controls and 100 healthy subjects were tested for the presence of RF, ACPA and anti-RA33 IgG/A/M isotypes by $\mathrm{EliA}^{\mathrm{TM}}$ (Thermo Fisher Scientific). RF and ACPA were routinely measured by nephelometry and the anti-CCP EliA ${ }^{\mathrm{TM}}$, respectively. For finding associations with American College of Rheumatology (ACR)20 and simplified disease activity score (SDAI)50 therapeutic responses, an inception cohort of 165 RA patients was analysed.

Results Diagnostic specificity of antibodies was at least 95\%. $185(63.4 \%)$ of $290 \mathrm{RA}$ patients tested positive for at least one routine marker (RF or ACPA) while 107 were negative for both antibodies (seronegative). Among these, 24 (8.2\%) patients tested positive for IgG/A/M anti-RA33 and/or IgA-RF/ ACPA. To determine the prognostic value regarding therapeutic responses a cross-validated combined model with an accuracy of $77 \%$ and an estimated $p$-value $(\mathrm{k}=10)$ of 0.00034 showed high levels $(>133 \mathrm{IU} / \mathrm{ml}$ ) of IgM-RF to be associated with a favourable response to methotrexate (MTX). In case of low or no RF, the presence of IgG-RA33 antibody on the one hand, and the absence of IgA-ACPA on the other hand was associated with a favourable response. 